\title{
Uniform Local Binary Pattern for Fingerprint Liveness Detection in the Gaussian Pyramid
}

\author{
Yujia Jiang (iD) and Xin Liu \\ College of Architecture and Artistic Design, Hunan Institute of Technology, Hengyang 421001, China \\ Correspondence should be addressed to Yujia Jiang; jiangyujiacaad@163.com
}

Received 11 July 2017; Revised 2 October 2017; Accepted 15 November 2017; Published 9 January 2018

Academic Editor: William Sandham

Copyright (C) 2018 Yujia Jiang and Xin Liu. This is an open access article distributed under the Creative Commons Attribution License, which permits unrestricted use, distribution, and reproduction in any medium, provided the original work is properly cited.

\begin{abstract}
Fingerprint recognition schemas are widely used in our daily life, such as Door Security, Identification, and Phone Verification. However, the existing problem is that fingerprint recognition systems are easily tricked by fake fingerprints for collaboration. Therefore, designing a fingerprint liveness detection module in fingerprint recognition systems is necessary. To solve the above problem and discriminate true fingerprint from fake ones, a novel software-based liveness detection approach using uniform local binary pattern (ULBP) in spatial pyramid is applied to recognize fingerprint liveness in this paper. Firstly, preprocessing operation for each fingerprint is necessary. Then, to solve image rotation and scale invariance, three-layer spatial pyramids of fingerprints are introduced in this paper. Next, texture information for three layers spatial pyramids is described by using uniform local binary pattern to extract features of given fingerprints. The accuracy of our proposed method has been compared with several stateof-the-art methods in fingerprint liveness detection. Experiments based on standard databases, taken from Liveness Detection Competition 2013 composed of four different fingerprint sensors, have been carried out. Finally, classifier model based on extracted features is trained using SVM classifier. Experimental results present that our proposed method can achieve high recognition accuracy compared with other methods.
\end{abstract}

\section{Introduction}

With the widespread use of smart applications and phones, it brings convenience to our life. However, the security of identity authentication is an issue that needs to be addressed urgently. Traditional identity authentication method can deal with the security issue, but current problem is that tokens or passwords are easy to be forgotten for a long time and bank cards or identity cards, and so on, are easily stolen or lost. Therefore, recognition systems based on biometric traits gradually replace traditional authentication methods nowadays. For example, smart phone fingerprint recognition device: the personal fingerprint information is stored on the phone, so only the user can access and turn on the smart phone. Biometrics system refers to the identification of human beings based on their physiological and behavioral characteristics. The ease of use and low error rates which promote their widespread use are superior to others methods.
Of course, biometric systems have their own weakness. For example, biometric systems contain vulnerabilities and are also susceptible to various kinds of sophisticated forms of spoofing. Among these, the fingerprint recognition, the ease of use, and high recognition rate are the main factors that contribute to their widespread use, accounting for the vast majority part [1]. Indeed, early identification systems can be easily spoofed by fake fingerprints, which can be reproduced from common materials. Popular fake fingertip materials such as silicon, wood glue and latex $[2,3]$ consist of large organic molecules which tend to agglomerate during processing. For example, threats to fingerprint systems are spoof finger attack at the sensor, attack on software modules, and so on [4].

Fingerprint liveness detection refers to whether the fingerprints presented are really from a live fingertip or spoofed ones. In order to solve the spoofing, various methods are proposed [5-8] in recent years. The goal of current fingerprint liveness detection research is concerned about how to design 
a method to discriminate real and fake fingerprints. Because the features of image can be described using textures information, many features based on texture extraction methods spring out according to human vision. In this paper, a novel fingerprint liveness detection method based on uniform local binary pattern in Gaussian pyramid has been proposed. Multiscale analysis using two layers Gaussian pyramid filter has been proved to be more efficient for solving image scale invariance. On the whole, fingerprint liveness detection is regarded as a binary classification problem, in which the given fingerprint image is either a real fingerprint or a spoof one. Feature extraction is a key step for solving the above problem. In our method, Gaussian pyramid filter is introduced to deal with the problem of scale invariance, and feature vectors are constructed using uniform local binary pattern to reduce the number of dimensionality of features. After these, feature vectors of each layer of spatial pyramid image are extracted through using uniform local binary pattern (ULBP). Once the feature vector is generated, the samples classifier model is learnt by using support vector machine. Experimental results based on the LivDet 2013 show that our method exhibits a strong edge.

The paper is organized as follows. In Section 2, a summary of the related work to the present research is given. Our proposed method about the feature vector extraction is introduced in Section 3. The result and comparison are given in Section 4. Conclusions are drawn in Section 5.

\section{Related Work}

Fingerprint authentication systems are easily cheated by these fake fingerprints, which fake fingerprints that mimic real users' fingerprints. Therefore, fingerprint liveness detection methods are proposed to prevent fingerprint authentication systems from being fake fingerprints. Because of the alternation of the ridges and valleys on them, fingertips surfaces are intrinsically coarse at certain scale. Furthermore, considering the advantage of software-based detection compared with the hardware-based method, software-based fingerprint liveness detection method is proposed in this paper. Because of moisture of sweat glands, the real fingerprint taken using fingerprint sensor devices will change slightly in a short time span. However, it will not happen in spoof ones. Therefore, researchers detect the fingerprint vitality through the analysis perspiration of fingerprint in different time, and the perspiration pattern changes at different time interval were observed (2 seconds in [6] and 5 seconds in [7]). In [7], they can find that the intervals are longer and the wavy nature is more complex based on the spreading of moisture for the real fingerprint. In order to improve the accuracy of Derakhshani's proposed method, Schuckers et al. [8, 9] proposed a novel liveness detection method by using wavelet analysis. In their method, multiresolution analyses are used to extract the low frequency content and wavelet packet analyses are used to extract the high frequency content. Other features related to spectral energy distribution have been used by using different transformation. Thin-plate model has been used by analysing fingertip distortion in [10]. In their method, the tester press his fingertip on the surface of fingerprint sensor and rotate their fingers in four directions ( 0 degree, 90 degree, 180 degree and 270 degree) to capture a sequence of frames. Then, relevant features based on the skin deformation based on capturing finger distortion images are constructed. In [11], a liveness detection method using one-way analysis of variance ANOVA and Multiple-Comparison Method to do the statistical tests on the dataset of real fingers and spoof ones was proposed. Because fake fingerprint image quality is not as good as the real fingerprint image, it is difficult to forge a real fingerprint image with the same or better quality fingerprint images. Based on this consideration, [12] uses the coarseness of the fingerprint to discriminate features. Nikam and Agarwal detected the fingerprint liveness based on ridgelet transform to extract texture features using only single fingerprint image [13]. The same method is researched in [14], where 25 quality parameters are used as the measure of performance detection. These methods are such as in $[15,16]$, in which the features used in the classifier are based on the specific fingerprint measurements, such as ridge strength, continuity, and clarity. Ghiani et al. [16] proposed a fingerprint liveness detection method based on wavelet analysis. In 2013, Ghiani et al. [17] detected the vitality of fingerprint images based on residual Gaussian and the noise of the fingerprint images to estimate the coarseness of fingerprint image. There are many small pores of circular structures in real fingertips, and the attackers observed that we cannot accurately imitate sweat pores in spoofed ones. Reference [18] proposed a new detection method by comparing pore quantity between recorded fingerprints and the query ones. After that, Gragnaniello et al. [19] proposed a novel detection method based on the optimum threshold from correlations peaks to detect which peaks are active pores. Many methods based on texture features have been proposed for liveness detection, such as statistical, signal processing approaches and model based and structural ones [20]. Rao and Jain [21] proposed a fingerprint liveness detection method based on minimizing the energy associated with phase and orientation maps. Multiresolution texture feature analysis in their method and cross ridge frequency analysis techniques are used. Ratha et al. [22] texture features based on the spatial gray level dependence method are extracted by using spatial gray level dependence method (SGLDM) for personal verification and discrimination. In latest literatures, much attention has been also focused on the wavelet transform domain. Jain et al. proposed many fingerprint liveness detection methods, such as the curvelet transform $[23,24]$, the Gabor filters [12], and the gray level cooccurrence matrices which are combined with the wavelet transform $[7,14,25]$.

\section{Feature Extraction}

The problem of fingerprint liveness detection is seen as twoclass classification problem where a given fingerprint image is either real or fake, so the feature extraction is the key step. A general diagram showing different phases of our method is shown in Figure 1, which mainly includes two phases: image training process and image testing process. Next we will give a detailed description on feature extraction of a given image. 


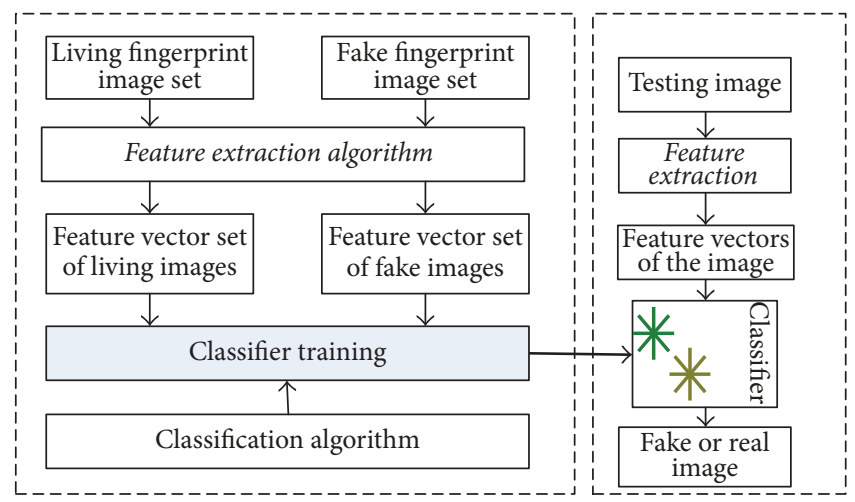

Figure 1: The flowchart of the proposed method [9].

3.1. Gaussian Pyramid Filter (GPF). Image pyramid is the major description of image multiscale, and it is an effective but simple structure that interprets image in multiple resolutions. An image pyramid is image set, which comes from the same original image and the resolutions of the image gradually decreases in the form of pyramid. It is sampled by a cascade downsampling until a certain termination condition is researched. We compare a layer of images to a pyramid; the higher the hierarchy, the smaller the image and the lower the resolution. An image pyramid is a series of images sets that are progressively reduced in the shape of a pyramid. At the bottom of the pyramid is the high-resolution, and the top pyramid is low resolution approximation. When moving to the top of the pyramid, the resolutions of images decrease gradually. Because of the size of the base $J$ is $2^{J} \times 2^{J}$ or $N \times N$, where $J=\log 2 N$; Thus, the size of intermediate $j$ is $2^{j} \times 2^{j}$, and the range of $j$ is $0 \leq j \leq J$. The complete pyramid is composed of $J+1$ resolutions, so the range of size of different resolutions is $2^{J} \times 2^{J}$ to $2^{0} \times 2^{0}$. However, most pyramids only have $P+1$ layers, where $1 \leq P \leq J$. In this paper, Gaussian pyramid is introduced to deal with scale invariance. Two steps are included: (a) the convolution of original Image $G_{i}$ and Gaussian kernel $K$ are conducted; (b) removing the even rows and columns using downsampling operation. Then the images shrunk to a quarter of the original image. Finally, We can obtain the entire pyramid by iterating over the steps above. The Gaussian kernel $K$ is as follows:

$$
K=\frac{1}{16} *\left[\begin{array}{ccccc}
1 & 4 & 6 & 4 & 1 \\
4 & 16 & 24 & 16 & 4 \\
6 & 24 & 36 & 24 & 6 \\
4 & 16 & 24 & 16 & 4 \\
1 & 4 & 6 & 4 & 1
\end{array}\right]
$$

After downsampling operation, the image is scaled to half of the original image. Figure 2(a) shows the original fingerprint image, and Figure 2(b) is an image after one-layer Gaussian kernel operation, and Figure 2(c) is an image after two-layer Gaussian kernel operation.
3.2. Uniform Local Binary Pattern. Local Binary Pattern (LBP), which has scale invariance and gray invariance, is used to describe the image local texture information, and the process of texture features extraction of given image is as follows. Firstly, the pixel values of eight neighbors in $3 \times 3$ window are compared with the center pixel of the $3 \times 3$ window, respectively, and binary relations are computed by using (2). In (2), $z$ denotes the comparative results between the center pixel and eight adjacent pixels, respectively. Then, the LBP code is weighted by using binary relationship of power of two and summed to obtain the LBP code of the current selected center pixel. Eq. (3) can calculate the LBP code. In (3), I $\left(x_{c}, y_{c}\right)$ represents the center pixel values of the $3 \times 3$ window, and the eight adjacent pixels are $g_{0}, \ldots, g_{7}$ :

$$
\begin{array}{r}
s(z)= \begin{cases}1, & z \geq 0 \\
0, & z<0,\end{cases} \\
\operatorname{LBP}\left(x_{c}, y_{c}\right)=\sum_{i=0}^{7} s\left(g_{i}, g_{c}\right) 2^{i} .
\end{array}
$$

An example of LBP operation is shown in Figure 3. Firstly, Figure $3(\mathrm{a})$ is the part of image, whose size is $3 \times 3$. In the $3 \times 3$ window, the pixel value of center pixel is 45 . Then, binary relationships are constructed using (2), in which eight adjacent pixel values are compared with the center pixel, respectively. The so-threshold binary values in Figure 3(b) responding to Figure 3(a) using (2) are obtained. Figure 3(c) is a binary relationship of power of two. Next we can calculate, converting the binary number into a decimal number in a clockwise direction by (3), the pixel value of center pixel using LBP. Finally, the local texture feature of image can be shown by counting the histogram of pixel value after LBP operation. Figure 3(c), LBP code of the center pixel: $1 \times 2^{0}+0 \times 2^{1}+0 \times$ $2^{2}+1 \times 2^{3}+0 \times 2^{4}+1 \times 2^{5}+1 \times 2^{6}+0 \times 2^{7}=1+8+32+64=105$.

Therefore, features of image can be described by counting histogram which is formed by 256 LBP codes. However, the current problem is that the binary models after LBP operation are too many. Thus, to solve the problem and improve statistics, uniform local binary pattern is proposed. 


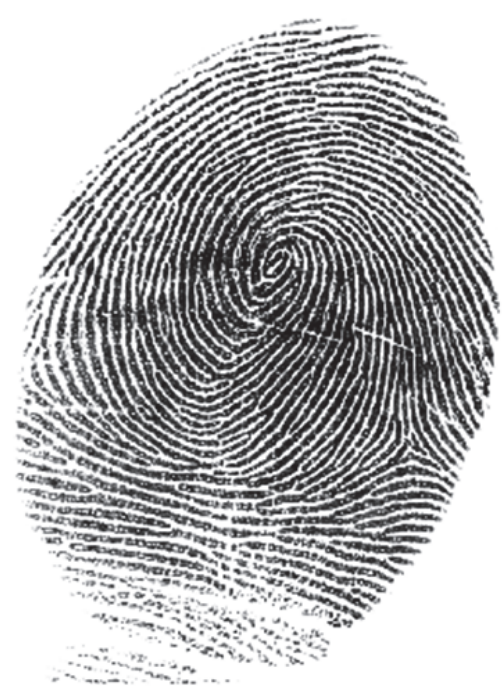

(a)

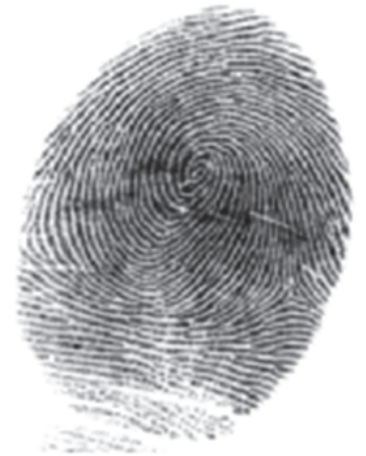

(b)

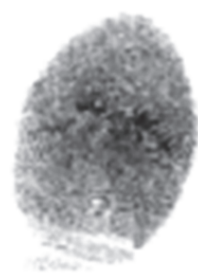

(c)

Figure 2: The different scale image based downsampling operation.

\begin{tabular}{|l|l|l|}
\hline 66 & 22 & 44 \\
\hline 23 & 45 & 80 \\
\hline 87 & 58 & 35 \\
\hline
\end{tabular}

(a)

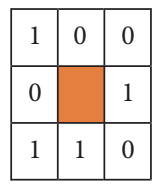

(b)

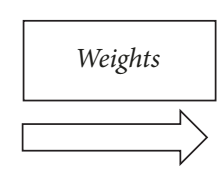

\begin{tabular}{|l|l|l|}
\hline $1 \times 2^{0}$ & $0 \times 2^{1}$ & $0 \times 2^{2}$ \\
\hline $0 \times 2^{7}$ & & $1 \times 2^{3}$ \\
\hline $1 \times 2^{6}$ & $1 \times 2^{5}$ & $0 \times 2^{4}$ \\
\hline
\end{tabular}

(c)

FIgURE 3: An example of the LBP operation.

It finds that the majority transformation of LBP modes only contains two jumps from one to zero or from zero to one, for example, 00000000 (zero jump), 00000111 (only one jump from zero to one), and 10001111 (two jumps are included, one is from one to zero, and another is from zero to one). The above three examples are all uniform local binary pattern. Besides these, others are called mixed model. Based on this finding, uniform local binary pattern is proposed. And for eight adjacent pixel sampling point in the $3 \times 3$ window, the number of original binary model is reduced from $2^{8}=256$ to $8 \times(8-1)+2=58$ after introducing uniform local binary pattern operation. On one hand, uniform local binary pattern can reduce the dimensionality of feature vectors, on the other hand, it can solve the effects of high frequency noise.

3.3. Support Vector Machine (SVM). Support Vector Machines (SVM) is a general two-class classifier, which is widely used in the research field of computer vision, pattern recognition, and deep learning. The goal of SVM is to find the hyperplane of the classification requirement, which can divide two classification samples correctly; at the same time, the classification interval is the largest. Figure 4 shows the optimal line of classification in linear separable cases. $H$ denotes the optimal line, which can be achieved by maximizing the distance $\max _{\text {margin }=w / 2}$ between $H_{1}$ and $H_{2}$. LIBSVM software package $[7,25,26]$ is a most commonly used classification tool. Two key issues need to be considered when using SVM.

One problem is how to select an appropriate kernel function of SVM. The goal of kernel function is that the two classification samples are linearly separable by an appropriate transformation in high-dimensional space; however, the two classification samples are linearly nonseparable in low dimensional space. According to the problem of linear separable and linear inseparable, different kernel functions are chosen. It is noted that the classification labels and features are all nonlinear. In our method, RBF kernel function is selected, which can make nonlinearly mapping to a high-dimensional space.

Another problem is how to select parameters. Two parameters are included in the RBF kernel function: $C$ and $\Upsilon$. To train a better classifier, parameter optimization operation is used. In LIBSVM, gnuplot.exe is an executable tool, which is used to find the optimal parameters pairs $C$ and $\Upsilon$, while the goal of the parameter optimization operation is to classify the unknown data. Finally, we can search the results of the optimal parameters by using the "Grid-search and Crossvalidation".

\section{Experiment}

The performance of our method is evaluated by using one official dataset: LivDet 2013 [27], which are the publicly 


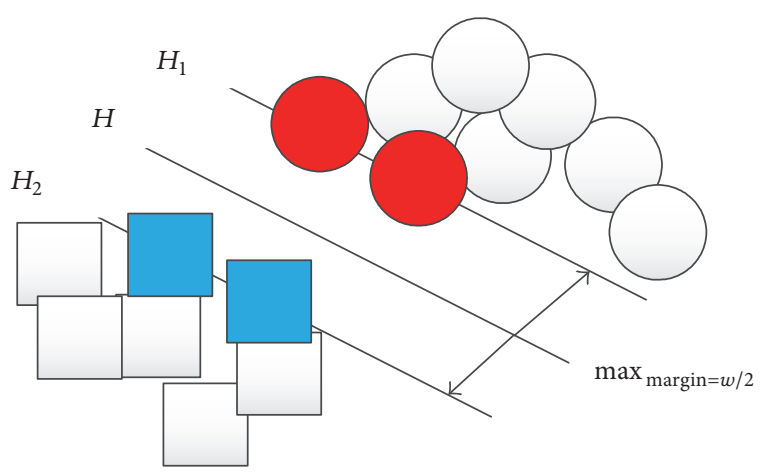

FIGURE 4: The optimal line of classification in linear separable cases.

available datasets provided in the 2013 Fingerprint Liveness Detection Competition. Firstly, a brief introduction about the official databases is given in this section. Secondly, feature vectors are constructed by using our method. Then, the validation criterion is defined which is used to evaluate the performance of our method. Finally, experiments results based on the official dataset LivDet 2013 are obtained, which shows that our proposed method is superior to others' methods.

4.1. Databases Introduction. The Department of Electrical and Computer Engineering of Clarkson University (USA) and the Department of Electronic Engineering of the University of Cagliari (Italy) held the first LivDet competition $[5,27]$ to help researchers study fingerprints liveness and compare the performance of the proposed state-of-the-art detection methods in 2009. Currently, four publicly official fingerprints datasets are published. And the LivDet 2013 Fingerprint dataset was divided into two parts: one is training part, which is used to learn a trained model classifier, and another set is testing samples, which are used to estimate the performance of the proposed method.

LivDet 2013 fingerprints are obtained through using four different optical fingerprint sensors (such as (a) Italdata ET10 (500 dpi), (b) CrossMatch Verifier 300LC (500 dpi), (c) Biometrika FX2000 (569 dpi), and (d) Swipe (96 dpi)). In this fingerprint set, 8874 real fingerprints and 7979 spoof fingerprints are included, and five different materials, such as Gelatin, Ecoflex, Latex, Modasil, and wood glue, are made fake fingerprints. A part of the fingerprints is used to train the SVM classifiers with the RBF kernel and the rest are used to evaluate the performance of classifier.

The dataset of each fingerprint sensor is divided into two parts: (1) a testing set and (2) a training set. As shown in Table 1, the fingerprint testing set is used to assess the performance of proposed method and the latter is used to learn a classifier model. More detailed parameters about LivDet 2013 are listed in Table 1. In Addition, some fingerprint images of real and spoof are presented in Figure 5. The material used is specified for the generation of the fake fingers, such as Playdoh, Latex, BodyDouble, and Ecoflex. It is hard for our eyes to distinguish the real fingerprints from the spoof ones. Therefore, our task is designing a method to distinguish the fingerprint liveness.

4.2. Gaussian Multiscale Transformation and Feature Extraction. In this experiment, to solve scale invariance of fingerprint image, Gaussian pyramid structure is used in the fingerprint liveness detection. After Gaussian pyramid operation, features based on uniform local binary pattern are extracted. For example, in Figure 2, the size of the original image Figure 2(a) is $256 \times 256$. First of all, preprocessing operation is necessary for the given fingerprint images, such as image gray processing. Then, two-layer Gaussian pyramid transformation has been done to solve the image scale invariance by calculating the convolution operation of original fingerprint image and Gaussian filter. After every one-layer Gaussian pyramid transformation, the size of the current image is a quarter of the previous level. Figure 2(b) and Figure 2(c) show the images after Gaussian filter operation. The two images are represented by the symbol $G P_{1}$ and $G P_{2}$, respectively, whose sizes are $128 \times 128$ and $64 \times 64$. To make scale invariance of the extracted texture feature, characteristics of rotation invariance are used based on uniform local binary pattern. Next, features are constructed in original fingerprint image and two-layer pyramids. Since the LBP code can describe local micromode information of the original image, the local features of the fingerprint image can be represented by counting the histogram of pixel values after LBP operation. However, uniform local binary pattern only considers the eight-bit binary of pixel values, which contain two jumps from one to zero or from zero to one in the eight-bit binary. Due to fingerprint images being transformed to two-layer pyramid, feature vectors are extracted based on ULBP in original fingerprint image and two-pyramid image. After constructing the feature vector with uniform local binary pattern, normalization is necessary to unify the data scales. Finally, features are extracted. These features of training sets are trained a classifier model by using SVM, and features of testing sets evaluated the performance of classifier.

4.3. Performance Metrics and Classification Results. The LivDet 2013 Datasets derive from 2013 Fingerprint Liveness Detection Competition, and these fingerprints are downloaded from the website. The performance of method is validated by using the Average Classification Error (ACE), which is considered as standard metric for evaluation of the fingerprint liveness. It is defined as

$$
\mathrm{ACE}=\frac{(\mathrm{FAR}+\mathrm{FRR})}{2},
$$

where, in (4),

FAR

$=\frac{\text { Total Number Imposter Fingerprints Accepted as Genuine }}{\text { Total Number of Forgery Tests Performed }}$,

FRR

$=\frac{\text { Total Number Genuine Fingerprints Accepted as Imposter }}{\text { Total Number of Genuine Matching Tests Performed }}$. 


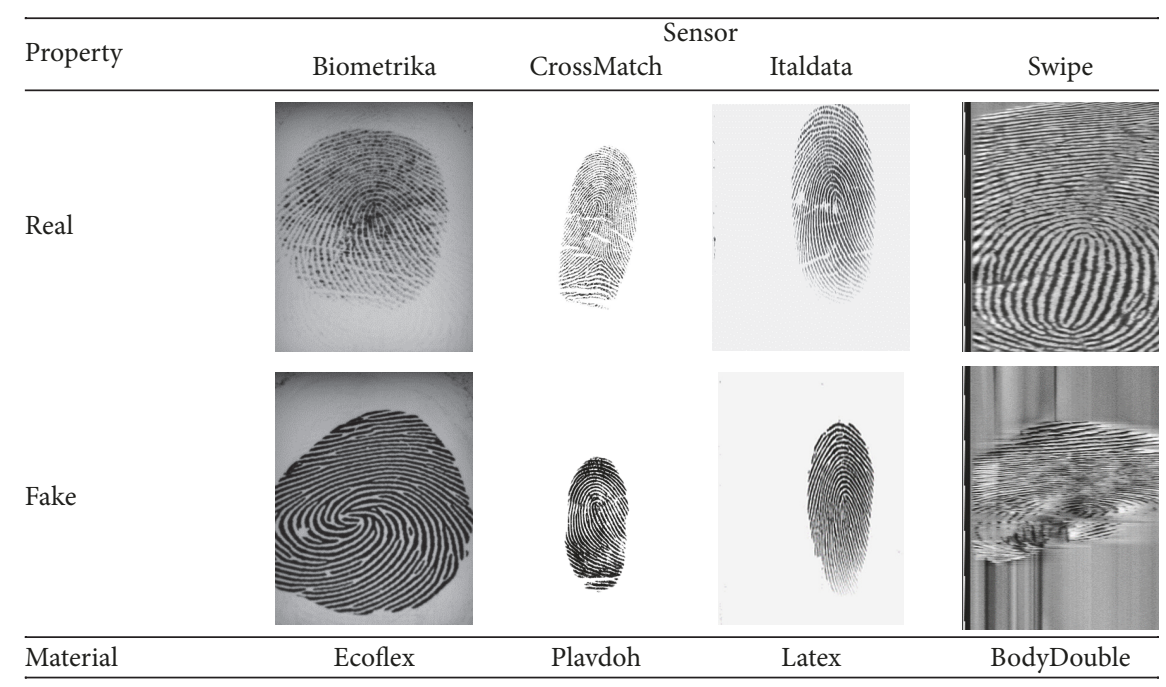

FIGURE 5: Typical sample images of real and spoof fingerprints those can be found in the LivDet 2013.

TABLE 1: Table of the detailed information of LivDet 2013.

\begin{tabular}{lcccccccc}
\hline \multirow{2}{*}{ Database ID } & Sensor & Model & number & Res. (dpi) & Image size & \multicolumn{2}{c}{$\begin{array}{c}\text { Number of image in } \\
\text { training set }\end{array}$} & \multicolumn{2}{c}{$\begin{array}{c}\text { Number of images in } \\
\text { testing set } \\
\end{array}$} & & & & & Real & Fake & Real & Fake \\
\hline Liv2013-1 & Biometrika & FX200 & 569 & $352 \times 384$ & 1000 & 1000 & 1000 \\
Liv2013-2 & CrossMatch & V300LC & 500 & $800 \times 750$ & 1250 & 1000 & 1250 & 1000 \\
Liv2013-3 & Italdata & ET10 & 500 & $480 \times 640$ & 1000 & 1000 & 1000 & 1000 \\
Liv2013-4 & Swipe & - & 96 & $1500 \times 208$ & 1221 & 979 & 1153 & 1000 \\
\hline
\end{tabular}

In (4), FAR (False Accept Rate) accounts for the percentage of fake fingerprints being misclassified as real one, and FRR (False Reject Rate) shows the percentage of real fingerprints being assigned to the spoofed ones. As shown in Figure 1, two successive processes are included: training process and testing processes.

Process 1 (training process). In this process, feature extraction is the key step to achieve fingerprint liveness detection. To solve the scale invariance and reduce the dimensionality of features, Gaussian pyramid in introduced to solve the problem of scale invariance and the uniform local binary pattern is used to reduce the dimensionality of feature. About the construction of feature vectors under different scale image having been talked in Section 4.2. Next, feature vector of original image and each pyramid image is extracted by using our proposed method. Finally, classifier is built by using executable file svm-train.exe to train the obtained feature vectors in SVM. In order to obtain optimal classifier, parameters optimization operation is necessary in this process. During the process, parameter pair $(C, \Upsilon)$ is learnt. Figure 6 shows optimal parameters pair values $(C, \Upsilon)$ for the four different fingerprint sensors. For example, the same color denotes the same value at the same $(C, \Upsilon)$. In Figure 6(a), the green line denotes the optimal parameters pairs when the value of parameter pair $(C, \Upsilon)$ is $(32768,8)$. And the classification accuracy is $95.5 \%$ in the current parameter pair $(C, \Upsilon)$. That is to say, we can obtain the best classifier model when parameter pair value is set as $(32768,8)$ in testing process. Similarly, the optimal parameter pairs corresponding Figures 5(b), 5(c), and $5(\mathrm{~d})$ can be found. If not operation, various of different parameter pairs $(C, \Upsilon)$ are needed to try to gain a better classifier model. Finally, the classifier has been trained.

Process 2 (testing process). To distinguish real fingerprint from the spoof ones, fingerprint liveness detection based on uniform local binary pattern in Gaussian pyramid is proposed in this paper. Before constructing feature vectors, image preprocessing operation is necessary. To address the scale invariance of images and reduce the dimensionality of features vectors, Gaussian pyramid filter operation is introduced. Both the Testing process and training processes are conducted based on MATLAB R2014a platform. As mentioned before, the executable file svm-train.exe tool are used to obtain the optimal parameter pair $(C, \Upsilon)$ in this paper. In our experiment, the ACE detection accuracy and its comparison with the current methods for detecting fingerprint vitality are listed in Table 2 . The accuracy of best designed methods from LivDet 2013 and the others' results are shown in Table 2. It shows that our method achieves detection 


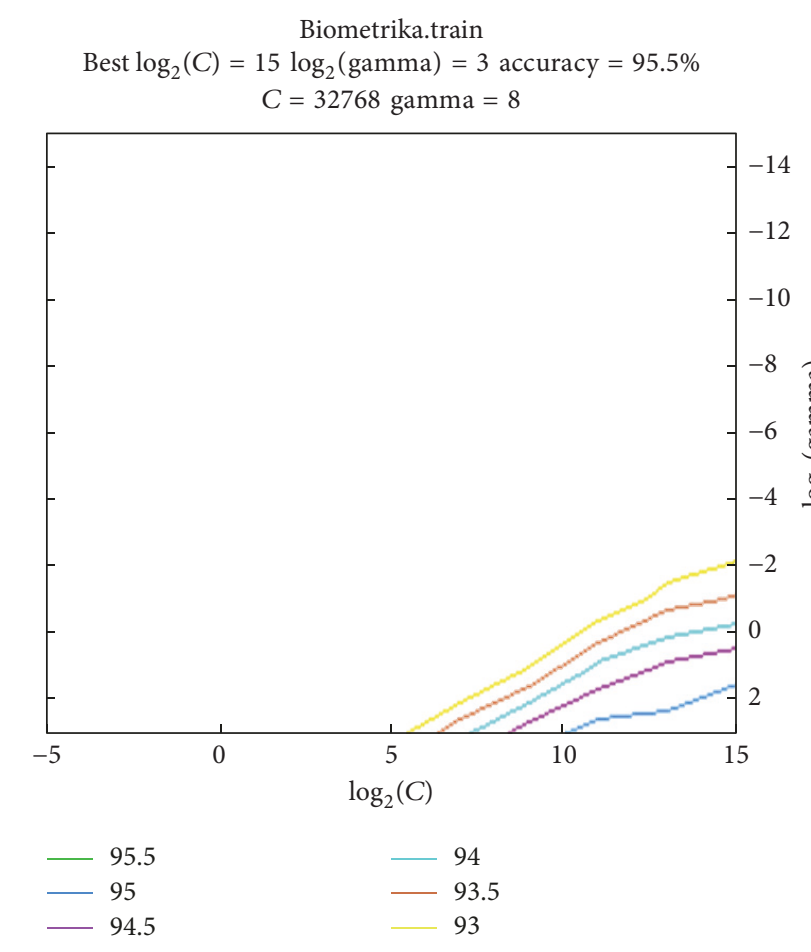

(a) Results of the Biometrika sensor

$$
\text { Italdata.train }
$$

Best $\log _{2}(C)=15 \log _{2}($ gamma $)=3$ accuracy $=95.7167 \%$

$$
C=32768 \text { gamma }=8
$$

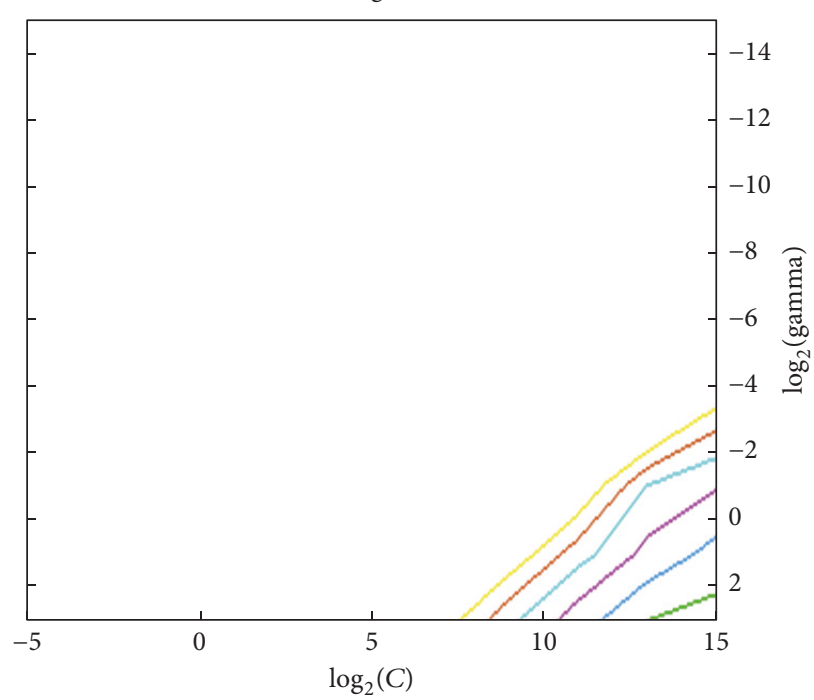

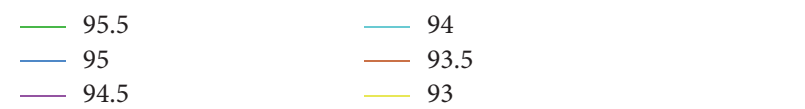

(c) Results of the Italdata sensor
CrossMatch.train

Best $\log _{2}(C)=15 \log _{2}($ gamma $)=3$ accuracy $=98.2519 \%$

$C=32768$ gamma $=8$

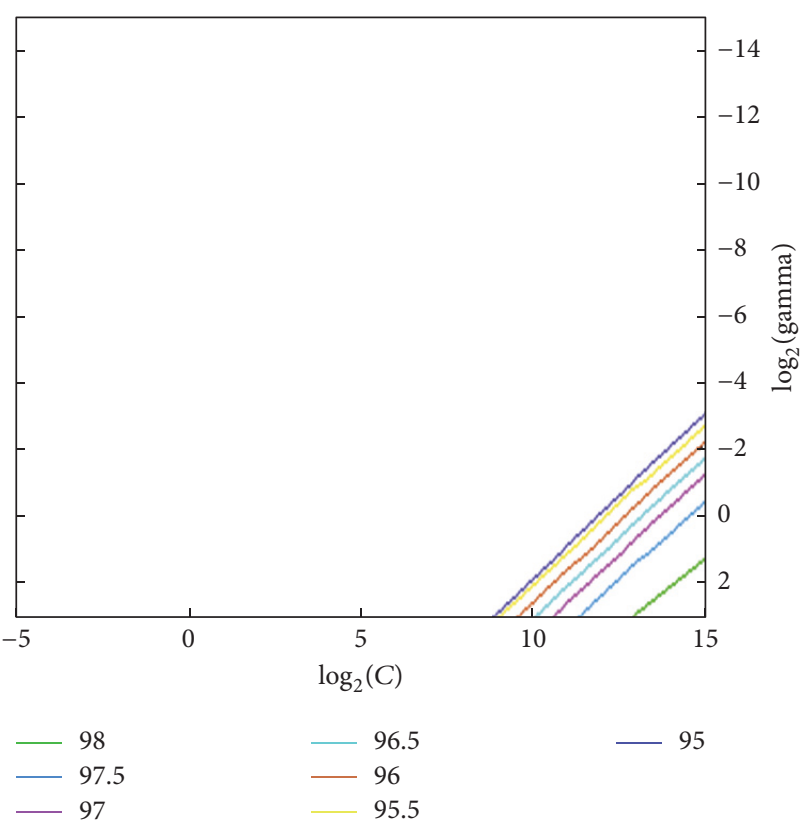

(b) Results of the CrossMatch sensor

Swipe.train

Best $\log _{2}(C)=15 \log _{2}($ gamma $)=1$ accuracy $=95.1667 \%$

$C=32768$ gamma $=2$

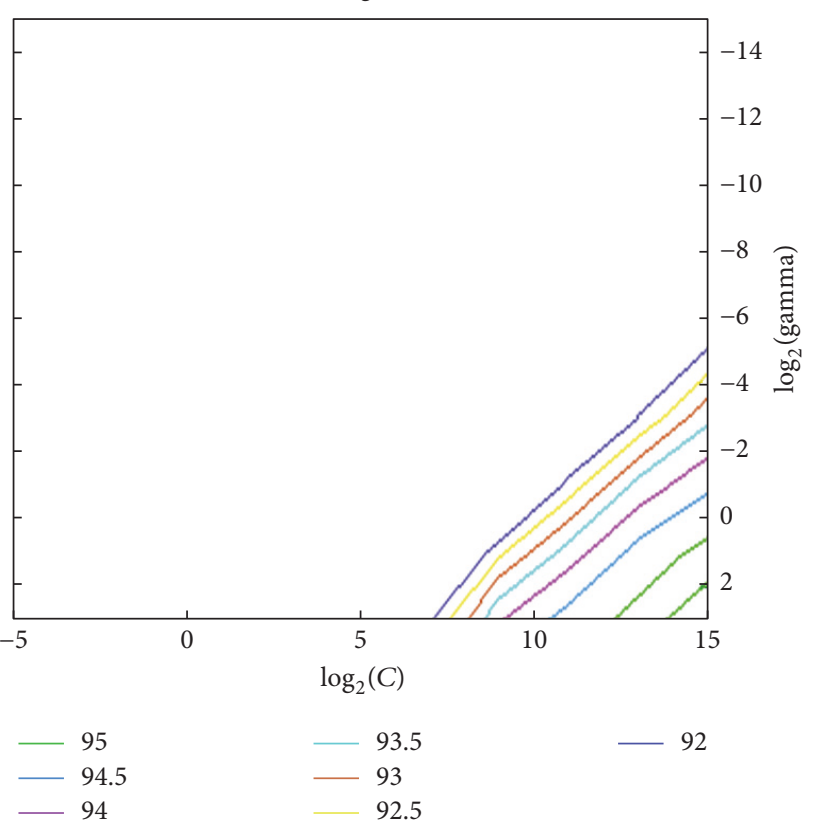

(d) Results of the Swipe sensor

FIgURE 6: Results of the Parameter optimization based on different sensors in LivDet 2013. 
TABLE 2: The results of the best different algorithms of LivDet 2013 in terms of average accuracy are cited from [27].

\begin{tabular}{lccccc}
\hline \multirow{2}{*}{ Methods } & & \multicolumn{2}{c}{ The average classification error ACE in (\%) } \\
& Bimometrika & Cmatch & Italata & Swipe & Average \\
\hline Our method & 10.68 & $\mathbf{4 6 . 0 9}$ & 13.7 & $\mathbf{2 1 . 2 0 5}$ \\
Frassetto2 [28] & 25.65 & 49.87 & 55.45 & $\mathbf{4 . 0 2}$ & 33.75 \\
ATVS [27] & $\mathbf{5 . 0 5}$ & 54.8 & 50 & 46.45 & 39.08 \\
UniNap2 [27] & 6.55 & 52.13 & $\mathbf{9 . 4 5}$ & 26.85 & 23.75 \\
HZ-JLW [27] & 32.95 & 55.56 & 13.15 & 15.19 & 29.21 \\
\hline
\end{tabular}

accuracy superior to other methods proposed in the LivDet 2013. In order to facilitate the comparison of the results, the best obtained results in Table 2 are highlighted in bold. We can clearly notice that the ACE (Average Classification Rate) of our method achieved is obviously superior to other ones in LivDet 2013.

\section{Conclusions and Future Work}

To solve fingerprint liveness and improve the security of authentication systems, a novel fingerprint liveness detection method based on uniform local binary pattern is proposed in this paper. The advantages of ULBP are that the dimensionality of feature vectors reduced compared with LBP. For the model of $3 \times 3$ window, the models of original eight bits binary reduce from 256 to 58 . The constructed feature vectors have been evaluated on publicly available databases LivDet 2013, which is a database released in the 2013 LivDet competition [27]. After the obtained features are trained by the SVM classifier, we can get a classifier model. With the help of the trained model, we can predict the classifier accuracy of testing dataset. Furthermore, the our method is part of the software-based solutions and it distinguishes real fingerprint images from the spoof ones; only one fingerprint without extra special hardware devices is added to the sensor. Liveness detection methods such as the one presented in this paper are of great importance in the biometric recognition field as they help to prevent direct attacks from the fake fingerprints (those carried out with synthetic traits and very difficult to detect).

The classifier performance of fingerprints datasets is extremely affected by the noise during the training process and testing process. When noise of fingerprint image is considered, the predicting results are unsatisfactory. Yet, we can get rid of the influence of noise by introducing noise filters with idea from Jin et al. [29]. Besides, more layers Gaussian pyramids are generated to further solve the scale invariance. These will be done in our future works.

\section{Conflicts of Interest}

The authors declare that they have no conflicts of interest.

\section{Acknowledgments}

This work was supported by the 12th Five-Year Programming of Scientific Research in Education of Hunan Province (Research on Paperless Test System of Art Design Course, no. XJK011CTM015) and Design and Study of Constructing Flipped Class Model Teaching Based On the Instructed Learning Plan + Microlecture (no. XJK015BGD018).

\section{References}

[1] D. Maltoni, D. Maio, A. Jain K, and S. Prabhakar, Handbook of Fingerprint Recognition, Springer Science Business Media, 2009.

[2] Z. Xia, R. Lv, Y. Zhu, P. Ji, H. Sun, and Y.-Q. Shi, "Fingerprint liveness detection using gradient-based texture features," Signal, Image and Video Processing, vol. 11, no. 2, pp. 381-388, 2017.

[3] J. Galbally, R. Cappelli, A. Lumini et al., "An evaluation of direct attacks using fake fingers generated from ISO templates," Pattern Recognition Letters, vol. 31, no. 8, pp. 725-732, 2010.

[4] N. K. Ratha, J. H. Connell, and R. M. Bolle, "Enhancing security and privacy in biometrics-based authentication systems," IBM Systems Journal, vol. 40, no. 3, pp. 614-634, 2001.

[5] D. Yambay, L. Ghiani, P. Denti, G. L. Marcialis, F. Roli, and S. Schuckers, "LivDet 2011 - Fingerprint liveness detection competition 2011," in Proceedings of the 2012 5th IAPR International Conference on Biometrics, ICB 2012, pp. 208-215, India, April 2012.

[6] S. T. V. Parthasaradhi, R. Derakhshani, L. A. Hornak, and S. A. C. Schuckers, "Time-series detection of perspiration as a liveness test in fingerprint devices," IEEE Transactions on Systems, Man, and Cybernetics, Part C: Applications and Reviews, vol. 35, no. 3, pp. 335-343, 2005.

[7] R. Derakhshani, S. A. C. Schuckers, L. A. Hornak, and L. O'Gorman, "Determination of vitality from a non-invasive biomedical measurement for use in fingerprint scanners," Pattern Recognition, vol. 36, no. 2, pp. 383-396, 2003.

[8] S. Schuckers and A. Abhyankar, "Detecting Liveness in Fingerprint Scanners Using Wavelets: Results of the Test Dataset," in Biometric Authentication, vol. 3087 of Lecture Notes in Computer Science, pp. 100-110, Springer, Berlin, Germany, 2004.

[9] Z. Xia, C. Yuan, X. Sun, R. Lv, D. Sun, and G. Gao, "Fingerprint liveness detection using difference co-occurrence matrix based texture features," International Journal of Multimedia and Ubiquitous Engineering, vol. 11, no. 11, pp. 1-16, 2016.

[10] P. Reddy V, A. Kumar, and S. Rahman, "A new anti-spoofing approach for biometric devices," IEEE Transactions on Biomedical Circuits Systems, vol. 2, no. 4, pp. 328-337, 2008.

[11] C. Sousedik and C. Busch, "Presentation attack detection methods for fingerprint recognition systems: a survey," IET Biometrics, vol. 3, no. 4, pp. 219-233, 2014.

[12] Y. S. Moon, J. S. Chen, K. C. Chan, K. So, and K. C. Woo, "Wavelet based fingerprint liveness detection," IEEE Electronics Letters, vol. 41, no. 20, pp. 1112-1113, 2005. 
[13] S. B. Nikam and S. Agarwal, "Ridgelet-based fake fingerprint detection," Neurocomputing, vol. 72, no. 10-12, pp. 2491-2506, 2009.

[14] Z. Xia, Y. Zhu, X. Sun, Z. Qin, and K. Ren, "Towards privacypreserving content-based image retrieval in cloud computing," IEEE Transactions on Information Forensics and Security, vol. PP, no. 99, 1 page, 2015.

[15] J. Galbally, F. Alonso-Fernandez, J. Fierrez, and J. OrtegaGarcia, "A high performance fingerprint liveness detection method based on quality related features," Future Generation Computer Systems, vol. 28, no. 1, pp. 311-321, 2012.

[16] L. Ghiani, G. L. Marcialis, and F. Roli, "Fingerprint liveness detection by local phase quantization," in Proceedings of the 21st International Conference on Pattern Recognition, ICPR 2012, pp. 537-540, November 2012.

[17] L. Ghiani, A. Hadid, G. L. Marcialis, and F. Roli, "Fingerprint Liveness Detection using Binarized Statistical Image Features," in Proceedings of the 6th IEEE International Conference on Biometrics: Theory, Applications and Systems, BTAS 2013, pp. 16, USA, October 2013.

[18] X. Jia, X. Yang, K. Cao et al., "Multi-scale local binary pattern with filters for spoof fingerprint detection," Information Sciences, vol. 268, pp. 91-102, 2014.

[19] D. Gragnaniello, G. Poggi, C. Sansone, and L. Verdoliva, "Fingerprint liveness detection based on Weber Local image Descriptor," in Proceedings of the 2013 4th IEEE Workshop on Biometric Measurements and Systems for Security and Medical Applications, BioMS 2013, Italy, September 2013.

[20] Y. Mei, H. Sun, and D. Xia, "A gradient-based combined method for the computation of fingerprints' orientation field," Image and Vision Computing, vol. 27, no. 8, pp. 1169-1177, 2009.

[21] A. R. Rao and R. C. Jain, "Computerized flow field analysis: oriented texture fields," IEEE Transactions on Pattern Analysis Machine Intelligence, vol. 14, no. 7, pp. 693-709, 1992.

[22] N. K. Ratha, S. Chen, and A. K. Jain, "Adaptive flow orientationbased feature extraction in fingerprint images," Pattern Recognition, vol. 28, no. 11, pp. 1657-1672, 1995.

[23] A. K. Jain, L. Hong, S. Pankanti, and R. Bolle, "An identityauthentication system using fingerprints," Proceedings of the IEEE, vol. 85, no. 9, pp. 1365-1388, 1997.

[24] H. H. Ahmed, H. M. Kelash, M. Tolba, and M. Badwy, "Fingerprint image enhancement based on threshold fast discrete curvelet transform (FDCT) and gabor filters," International Journal of Computer Applications, 2015.

[25] S. B. Nikam and S. Agarwal, "Fingerprint liveness detection using curvet energy and co-occurrence signatures," in Proceedings of the 5th International Conference on Computer Graphics, Imaging and Visualisation, Modern Techniques and Applications, CGIV, pp. 217-222, Malaysia, August 2008.

[26] Z. Xia, N. N. Xiong, A. V. Vasilakos, and X. Sun, "EPCBIR: An efficient and privacy-preserving content-based image retrieval scheme in cloud computing," Information Sciences, vol. 387, pp. 195-204, 2017.

[27] L. Ghiani, D. Yambay, V. Mura et al., "LivDet 2013 fingerprint liveness detection competition 2013," in Proceedings of the 6th IAPR International Conference on Biometrics, ICB 2013, Madrid, Spain, June 2013.

[28] R. Frassetto Nogueira, R. De Alencar Lotufo, and R. Campos Machado, "Evaluating software-based fingerprint liveness detection using Convolutional Networks and Local Binary Patterns," in Proceedings of the 2014 5th IEEE Workshop on
Biometric Measurements and Systems for Security and Medical Applications, BIOMS 2014, pp. 22-29, Italy, 2014.

[29] Q. Jin, I. Grama, and Q. Liu, "Optimal Weights Mixed Filter for removing mixture of Gaussian and impulse noises," PLOS ONE, vol. 12, no. 7, p. e0179051, 2017. 


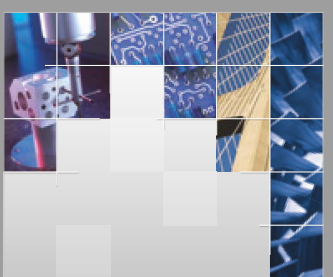

\section{Enfincering}
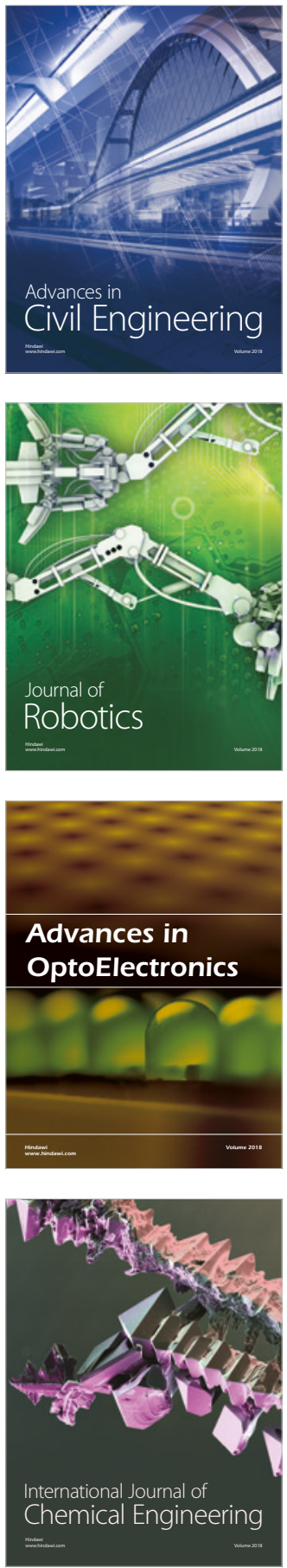

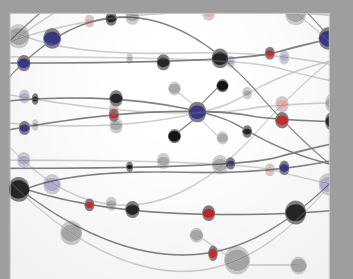

\section{Rotating \\ Machinery}

The Scientific World Journal

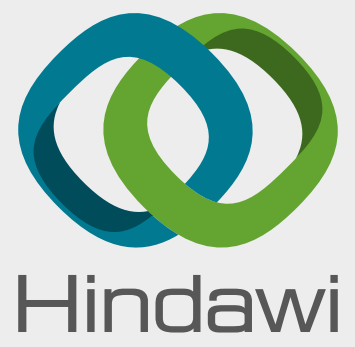

Submit your manuscripts at

www.hindawi.com
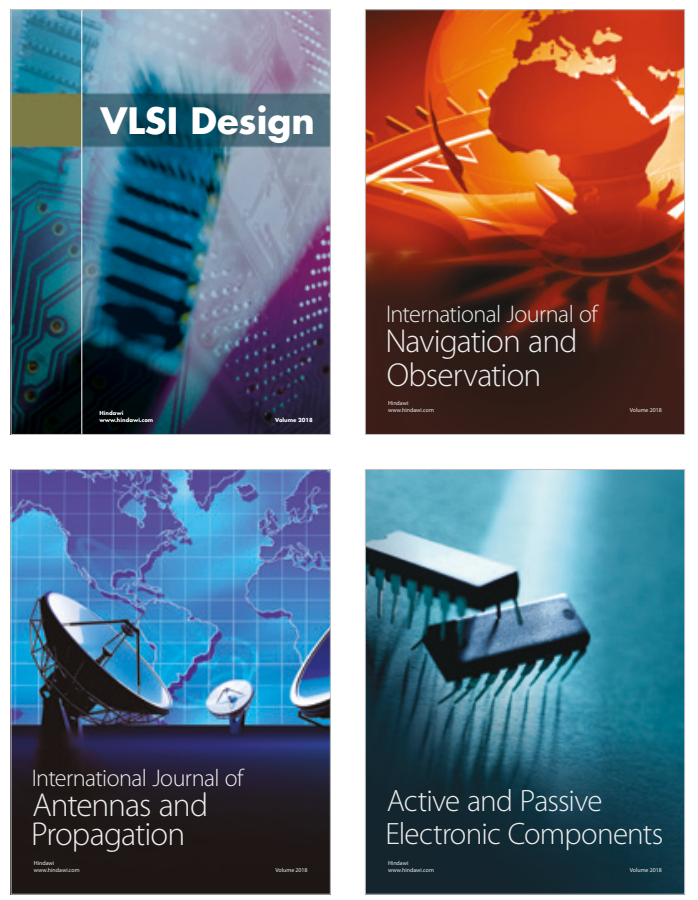
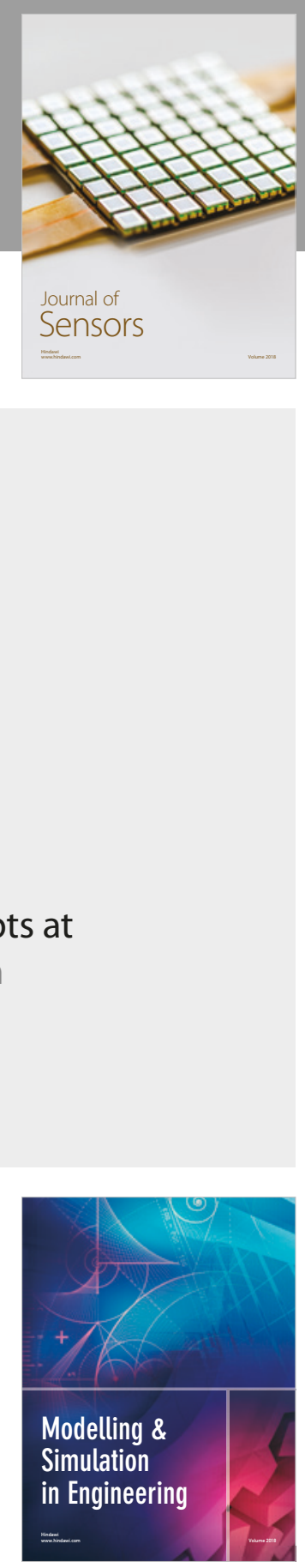

\section{Advances \\ Multimedia}
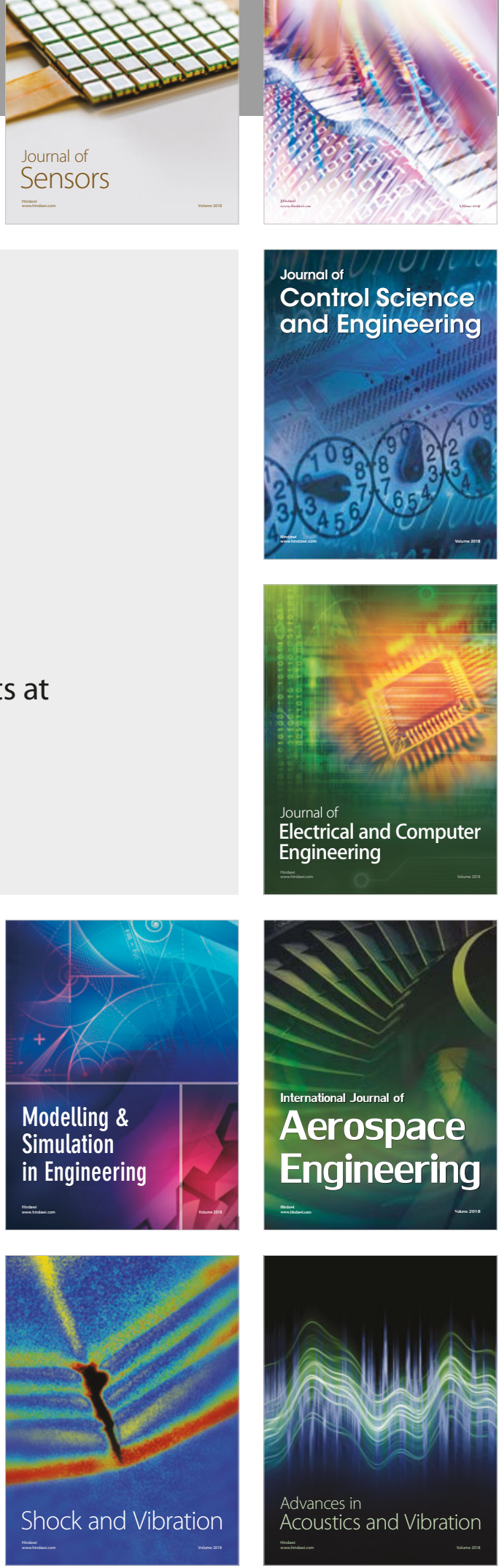